\title{
Sub-part-per-billion monitoring of nitric oxide by use of wavelength modulation spectroscopy in combination with a thermoelectrically cooled, continuous-wave quantum cascade laser
}

\author{
B. W. M. Moeskops, * S. M. Cristescu, and F. J. M. Harren \\ Department of Molecular and Laser Physics, Institute for Molecules and Materials, Radboud University Nijmegen, \\ P.O. Box 9010, NL-6500 GL Nijmegen, The Netherlands
}

\begin{abstract}
Received November 18, 2005; revised December 13, 2005; accepted December 13, 2005; posted December 19, 2005 (Doc. ID 66103)
We used a thermoelectrically cooled, continuous-wave, quantum cascade laser operating between 1847 and $1854 \mathrm{~cm}^{-1}$ in combination with wavelength modulation spectroscopy for the detection of nitric oxide (NO) at the sub-part-per-billion by volume (ppbv) level. The laser emission overlaps the $P_{7.5}$ doublet of NO centered around $1850.18 \mathrm{~cm}^{-1}$. Using an astigmatic multiple-pass absorption cell with an optical path length of $76 \mathrm{~m}$, we achieved a detection limit of $0.2 \mathrm{ppbv}$ at $10 \mathrm{kPa}$, with a total acquisition time of $30 \mathrm{~s}$. The corresponding minimal detectable absorption is $8.8 \times 10^{-9} \mathrm{~cm}^{-1} \mathrm{~Hz}^{-1 / 2}$. (c) 2006 Optical Society of America

OCIS codes: $280.1120,300.6360$.
\end{abstract}

Tunable infrared laser-based absorption spectroscopy is well suited for trace-gas sensing in different areas ranging from environmental monitoring ${ }^{1}$ to medical diagnostics, ${ }^{2}$ because it can deliver the sensitivity, specificity, and speed needed for such applications.

One of the most convenient light sources used is the quantum cascade laser (QCL), because of its compact design and easy operation, relatively high laser powers at the milliwatt level, and operation in the mid-infrared, where many molecules show strong absorption. A QCL has a typical tuning range of a few, inverse centimeters, which is relatively small compared with, for example, high-power, continuouswave (cw) optical parametric oscillators. On the other hand, the costs are significantly lower, which makes QCLs an attractive source for monitoring specific gases. Therefore, various spectroscopic methods using QCLs as a light source have been proposed during the past few years and applied for trace-gas sensing. These include frequency modulation spectroscopy, ${ }^{3}$ direct absorption spectroscopy using long-path multipass cells, ${ }^{4}$ photoacoustic spectroscopy ${ }^{5}$ cavity ringdown spectroscopy, ${ }^{6}$ cavityenhanced absorption spectroscopy, ${ }_{7-9}^{4}$ and off-axis integrated cavity output spectroscopy.

Only recently, cw QCLs that are able to operate in single mode at room temperature have become available. ${ }^{10}$ Single-mode operation is an important step forward for molecular spectroscopy, because of the narrow absorption lines down to tens of megahertz. Since they are thermo-electrically cooled, these single-mode cw QCLs do not require liquid nitrogen cooling and therefore are well suited to be utilized in a compact and high-sensitivity trace-gas sensor.

In this Letter we show the capability of the singlemode thermoelectrically cooled cw QCL for molecular absorption spectroscopy. The laser is used in combination with wavelength modulation spectroscopy for the detection of nitric oxide (NO) at and below the part-per-billion by volume (ppbv) mixing ratio. A schematic arrangement of the experimental setup is shown in Fig. 1. The QCL has a thermoelectrically cooled housing (Alpes Lasers); the laser is supplied by an external current driver (Keithley 2420). The QCL operates in the wavelength region between 1854 and $1847 \mathrm{~cm}^{-1}$ at an output power ranging from $0.5 \mathrm{~mW}$ at $273 \mathrm{~K}$ to $5 \mathrm{~mW}$ at $243 \mathrm{~K}$. The laser is scanned via a function generator and additional homemade electronics; the latter also serves as overvoltage protection for the laser.

For wavelength modulation spectroscopy the modulation depth is determined by optimizing the peak-to-trough values of the $2 f$ signal from the unre-

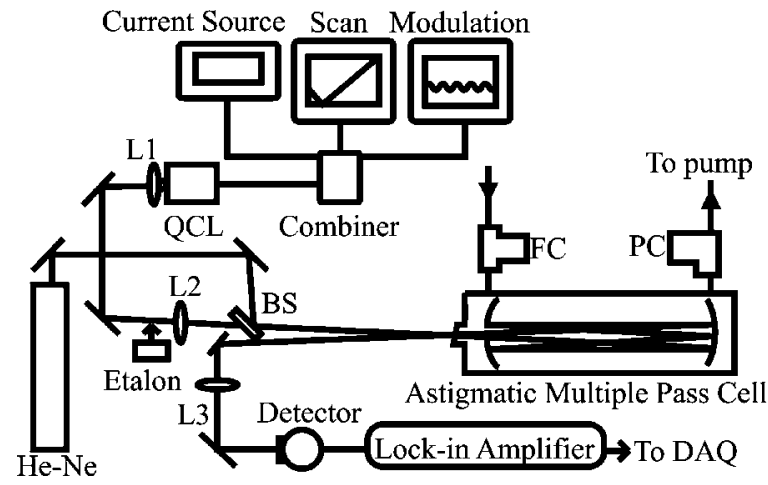

Fig. 1. Schematic diagram of the experimental setup: a combination of a stable current with scanning and modulating function generators form the laser driver electronics. Their signals are added in a homemade combiner that also acts as voltage protection for the QCL. A 0.5 in.-diameter ZnSe lens of $0.5 \mathrm{in}$. focal length (L1) collimates the beam before it is redirected via a $1 \mathrm{in}$. diameter $\mathrm{CaF}_{2}$ lens (L2) of $50 \mathrm{~cm}$ focal length to the astigmatic multiple-pass absorption cell. A He-Ne laser is used as a tracer beam and coupled in via a beam splitter (BS). After leaving the absorption cell, the light is focused by a $1 \mathrm{in}$. diameter $\mathrm{CaF}_{2}$ lens (L3) of $15 \mathrm{~cm}$ focal length onto a fast liquid nitrogen cooled $\mathrm{HgCdTe}$ detector. The $2 f$ signal from the lock-in amplifier is sent to a computer via a fast 14 bit digitizer for data acquisition (DAQ). PC, pressure controller; FC, flow controller. 


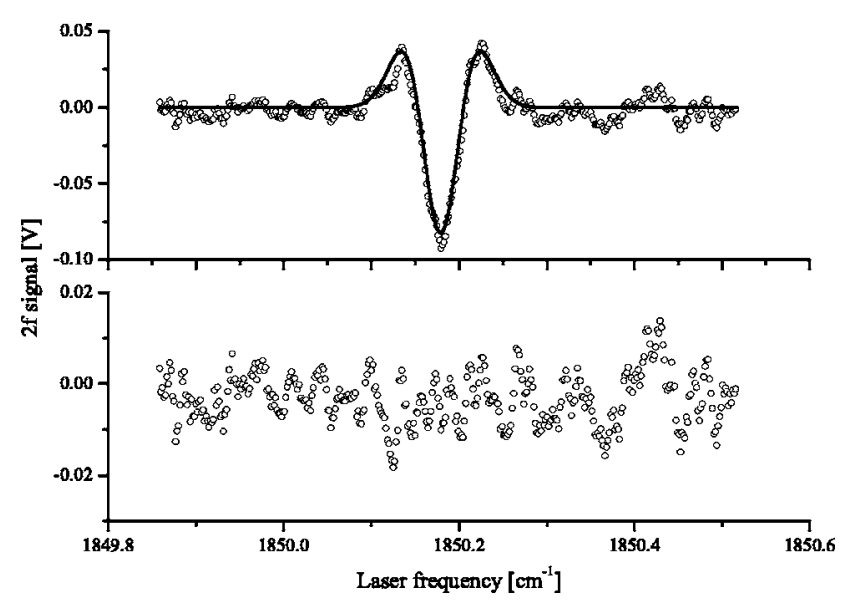

Fig. 2. NO absorption spectrum of 5 ppbv NO in $\mathrm{N}_{2}$ (top panel) after subtraction of the $\mathrm{N}_{2}$ background. Experimental data (circles) and the best fit (solid curve) are shown. For the fit we used a numerical approximation of the second derivative of a Voigt profile. The bottom panel shows the residue after subtraction of the fit.

solved NO-doublet at $1850.18 \mathrm{~cm}^{-1}{ }^{11}$ The modulation frequency $f$ was set to $250 \mathrm{kHz}$, and the $2 f$ signal was detected with a lock-in amplifier (SRS 844, Stanford Research Systems). The integration time of the lock-in amplifier was set to $100 \mu \mathrm{s}$, which allowed a wavelength scanning rate of $100 \mathrm{~Hz}$ over the selected wavelength range of the laser $\left(0.25 \mathrm{~cm}^{-1}\right)$ at a single thermoelectric temperature. The wavelength scans were stored using a fast data acquisition card (Gage Compuscope 14200). For detection of NO the laser beam was collimated using a short-focus ZnSe collimating lens of $0.5 \mathrm{in}$. ( $1 \mathrm{in} .=2.54 \mathrm{~cm})$ diameter and focal length and directed into a multiple-pass optical cell (Aerodyne, AMAC-76) with an absorption path length of $76 \mathrm{~m}$ and a volume of $300 \mathrm{ml}$. During flowthrough experiments the pressure inside the cell was kept at 100 mbars $(10$ mbars $=1 \mathrm{kPa})$ by a pressure controller and a pump (Fig. 1). After the absorption cell the laser beam was focused by a $\mathrm{CaF}_{2}$ lens of $15 \mathrm{~cm}$ focal length onto a fast, liquid nitrogen cooled detector (KV-104, Kolmar Technologies). All transmissive optics were positioned such that optical interference in the beam path was minimized.

Figure 2 shows the wavelength modulation signal obtained after averaging 2000 frequency scans (10 ms each) over the $P_{7.5}$ doublet of $\mathrm{NO}$ at $1850.18 \mathrm{~cm}^{-1}$, which has an absorption coefficient equal to $80 \%$ of the strongest NO doublet at $1903.13 \mathrm{~cm}^{-1}$. Here, the gas sample consisted of 5 ppbv $\left(1 \times 10^{-9}\right)$ NO in $\mathrm{N}_{2}$ at 100 mbars of pressure. After background subtraction, the absorption signal was fitted using a numerical approximation of the second derivative of a Voigt profile. Comparing the area of the Voigt curve with the standard deviation in the Gaussian distributed residual, we obtain an instrumental sensitivity of $0.2 \mathrm{ppbv}$ for a total acquisition time of $30 \mathrm{~s}$. Figure 3 shows the results from a dilution experiment in which the area of the fitted Voigt profile was taken as a measure of the NO concentration in the absorption cell. To determine the detection limit of our instrument, the standard devia- tion in the measured NO concentrations was calculated while the composition of the sample gas was kept constant. This resulted in a detection limit for NO of $0.2 \mathrm{ppbv}$ in $\mathrm{N}_{2}$ at a pressure of 100 mbar and an acquisition time of $30 \mathrm{~s}$. The corresponding minimal detectable absorption is $8.8 \times 10^{-9} \mathrm{~cm}^{-1} \mathrm{~Hz}^{-1 / 2}$ for an absorption path length of $76 \mathrm{~m}$.

It is generally accepted that air quality has an important effect on human health. Concern over the health damage caused by polluted air, particularly in urban areas, has risen in recent years. Among other compounds, NO is an important constituent of urban air pollution. Therefore, we decided to test the capabilities of our setup by sampling air from a nearby road. Atmospheric samples were taken at a $1 \mathrm{~m}$ distance from the road and at a height of $3 \mathrm{~m}$ via $40 \mathrm{~m}$ long Teflon tubing (inner diameter of $2 \mathrm{~mm}$ ) and directed to the laboratory setup at a flow rate of $20 \mathrm{l} / \mathrm{h}$. Water vapor was removed from the gas flow using $\mathrm{CaCl}_{2}$ to prevent spectroscopic interference with the NO absorption lines. ${ }^{11}$ The total gas handling system had a time delay of $30 \mathrm{~s}$; the absorption cell was refreshed every $5 \mathrm{~s}$. The passage of buses and heavy traffic near the sampling point resulted in large increases in the NO concentration (Fig. 4).

In conclusion, we have demonstrated that wavelength modulation spectroscopy in combination with a thermoelectrically cooled, cw QCL at $1850.18 \mathrm{~cm}^{-1}$ is suitable for detection of nitric oxide down to $0.2 \mathrm{ppbv}$ in $\mathrm{N}_{2}$ (30 $\mathrm{s}$ acquisition time), which is equivalent to a sensitivity of $8.8 \times 10^{-9} \mathrm{~cm}^{-1} \mathrm{~Hz}^{-1 / 2}$. We have developed a compact, convenient setup that allows measurements with high sensitivity, is relatively easy to align compared with cavity-enhanced techniques, and can be configured for autonomous operation. The sensitivity achieved here is limited mainly by residual interference between optical elements. Our detection limit for NO of 0.2 ppbv over $30 \mathrm{~s}$ can be compared with previous results of $0.12 \mathrm{ppbv} \mathrm{Hz}^{-1 / 2}$ obtained using a $210 \mathrm{~m}$ path length

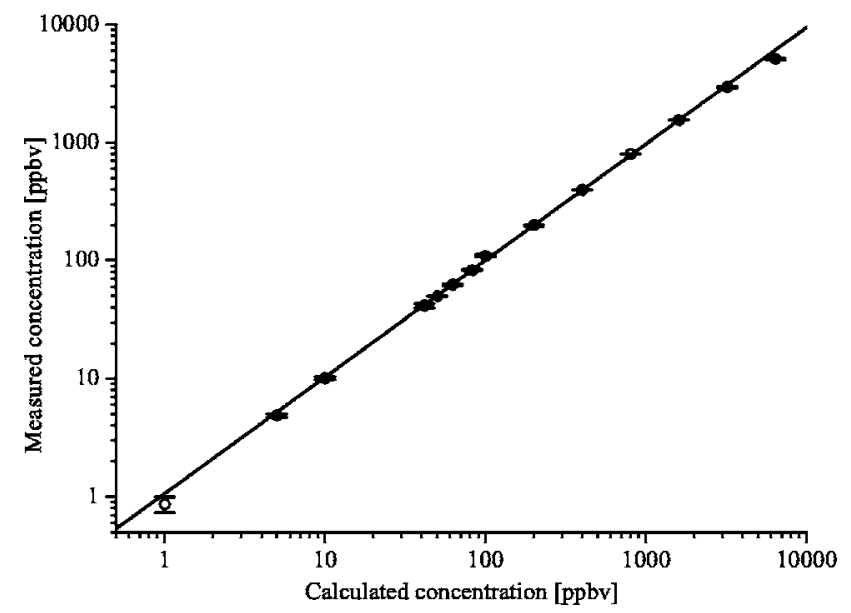

Fig. 3. Linearity of a measured NO concentration versus the diluted NO gas mixture in $\mathrm{N}_{2}\left(R^{2}=0.9995\right)$. The gases were dynamically mixed via mass flow controllers. The noise-equivalent NO concentration is $0.2 \mathrm{ppbv}$ with an acquisition time of $30 \mathrm{~s}$. 


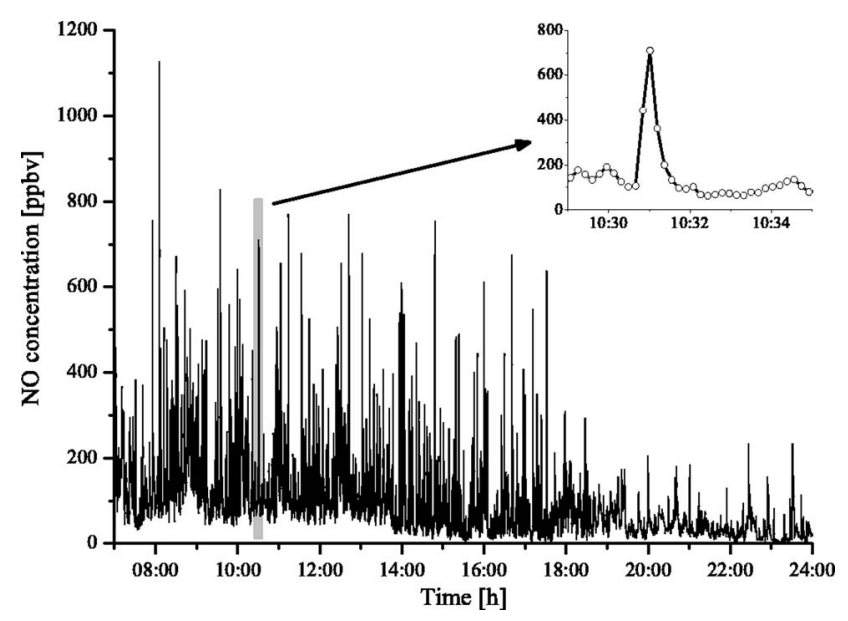

Fig. 4. NO concentration sampled on a normal weekday from a nearby roadside showing NO emissions by buses and heavy traffic. The inset shows a sharp rise in the NO concentration, associated with the passage of a single vehicle.

and temporal gating of signal and reference laser pulses on the detector, ${ }^{12} 41$ ppbv at 25 mbars using Faraday modulation spectroscopy, ${ }^{13} 0.7 \mathrm{ppbv}$ over $8 \mathrm{~s}$ at 80 mbars using cw cavity ringdown spectroscopy, ${ }^{6}$ and 1 ppbv for CO-laser based photoacoustics. ${ }^{14}$ For a liquid-nitrogen-free setup, Peltier-cooled detectors are available. The lower sensitivity of these detectors may be compensated for by the availability of cw QClasers with higher output power. Furthermore, tracegas detection using thermoelectrically cooled cw QCLs has proved its capability in environmental monitoring and shows promise for other applications in life sciences and medical diagnostics as well.

B. W. M. Moeskops's e-mail address is B.Moeskops @science.ru.nl.

*Visiting address, Toernooiveld 16525 ED Nijmegen, The Netherlands. Corresponding author, B. W. M. Moeskops; e-mail, B.Moeskops @science.ru.nl; fax, (+31)-24-3653311; telephone, (+31)-24-3653029.

\section{References}

1. P. A. Martin, Chem. Soc. Rev. 31, 201 (2002).

2. C. D. Mansfield, H. H. Mantsch, and H. N. Rutt, Can. J. Anal. Sci. Spectrosc. 47, 14 (2002).

3. K. Namjou, S. Cai, E. A. Whittaker, J. Faist, C. Gmachl, F. Capasso, D. L. Sivco, and A. Y. Cho, Opt. Lett. 23, 291 (1998).

4. L. Menzel, A. A. Kosterev, R. F. Curl, F. K. Tittel, C. Gmachl, D. L. Sivco, J. N. Baillargeon, A. L. Hutchinson, and A. Y. Cho, Appl. Phys. B 72, 859 (2001).

5. B. A. Paldus, T. G. Spence, R. N. Zare, J. Oomens, F. J. M. Harren, D. H. Parker, C. Gmachl, F. Capasso, D. L. Sivco, J. N. Baillargeon, A. L. Hutchinson, and A. Y. Cho, Opt. Lett. 24, 178 (1999).

6. A. A. Kosterev, A. L. Malinovsky, F. K. Tittel, C. Gmachl, F. Capasso, D. L. Sivco, J. N. Baillargeon, A. L. Hutchinson, and A. Y. Cho, Appl. Opt. 40, 5522 (2001).

7. J. B. Paul, J. J. Scherer, A. O'Keefe, L. Lapson, J. G. Anderson, C. Gmachl, F. Capasso, and A. Y. Cho, in Proc. SPIE 4577, 1 (2002).

8. Y. A. Bakhirkin, A. A. Kosterev, C. Roller, R. F. Curl, and F. K. Tittel, Appl. Opt. 43, 2257 (2004).

9. M. L. Silva, D. M. Sonnenfroh, D. I. Rosen, M. G. Allen, and A. O'Keefe, Appl. Phys. B 81, 705 (2005).

10. S. Blaser, D. A. Yarekha, L. Hvozdara, Y. Bonetti, A. Muller, M. Giovannini, and J. Faist, Appl. Phys. Lett. 86, 041109 (2005).

11. L. S. Rothman, A. Barbe, C. D. Brenner, L. R. Brown, C. Camy-Peyret, M. R. Carleer, K. Chance, C. Clerbaux, V. Dana, V. M. Devi, A. Fayt, J. M. Flaudi, R. R. Gamache, A. Goldman, D. Jacquemart, K. W. Jucks, W. J. Lafferty, J. Y. Mandin, S. T. Massie, V. Nemtchinov, D. A. Newnham, A. Perrin, C. P. Rinsland, J. Schroeder, K. M. Smith, M. A. H. Smith, K. Tang, R. A. Toth, J. Vander Auwera, P. Varanasi, and K. Yoshino, J. Quant. Spectrosc. Radiat. Transf. 82, 5 (2003).

12. D. D. Nelson, J. H. Shorter, J. B. McManus, and M. S. Zahniser, Appl. Phys. B 75, 343 (2002).

13. H. Ganser, W. Urban, and J. M. Brown, Mol. Phys. 101, 545 (2003).

14. L. A. J. Mur, I. E. Santosa, L. J. J. Laarhoven, N. J. Holton, F. J. M. Harren, and A. R. Smith, Plant Physiol. 138, 1247 (2005). 